\title{
Experimental and Numerical Research on the Formation Conditions and Entrainment Characteristics of Supercavity by Rear Jet Reflux
}

\author{
B. Liu, M. Xiang ${ }^{\dagger}$, X. Zhao, W. Zhang and J. Li \\ National University of Defense Technology, Changsha, Hunan, 410073, P.R. China \\ †Corresponding Author Email: xiangmin333@hotmail.com \\ (Received May 21, 2021; accepted September 5, 2021)
}

\begin{abstract}
Aiming at better controlling the ventilated supercavity flow for drag reduction, the experimental and numerical researches of supercavity by rear gas reflux are proposed in this paper. Several experiments with different test bodies have been carried out to study the formation and collapse conditions of jet-reflux supercavity. An opencirculation water tunnel for ultra-high-speed jet experiment and air jet system is employed to form jet-reflux supercavity around the bodies installed in the forward strut. The experiment results show that the supercavity can be maintained by the reflux of tail jet flow when an initial supercavity covering the jet outlet is formed. However, the supercavity will be destroyed when the jet intensity is further enhanced. Under the same jet coefficient, the scale of jet-reflux supercavity extends as the increase of the body length, while the critical jet coefficient for the collapse of the supercavity decreases as the increase of the body length. The multiphase flow model coupling the VOF model and the level-set method is applied to capture the air-water interface. Then, the flow field characteristics of the jet-reflux supercavity are analyzed and compared with the ventilated supercavity. The streamline inside the cavity presents considerable three-dimensional asymmetry inflating flow characteristics. The variation of the gas reflux coefficient along the axial direction is obtained, which indicates that a handful of reflux gas are required to sustain the head cavity. Therefore, the jet-reflux supercavity can be formed within a certain range of the tail jet intensity. Although jet intensities are not equal to each other, the scale of cavity head is roughly maintained under the same reflux coefficient. When the supercavity gets closed to the nozzle outlet, the maximum scale of cavity is decreased, which leads to a weaker reflux at the outlet. The cavity interface will be impinged by the high-speed gas and mixture liquid, which obviously causes deformation and final collapse. In order to improve the stability of the jet-reflux supercavity, it is necessary to use the gas re-directed structure to control the reflux.
\end{abstract}

Keywords: Supercavitation; Ventilated supercavity; Gas jet; Tail jet reflux; Entrainment; Water tunnel.

\section{NOMENCLATURE}

$\begin{array}{ll}d & \text { body diameter } \\ d_{c} & \text { cavitator diameter } \\ F r & \text { Froude numbers } \\ L_{c} & \text { length of cavity } \\ \bar{L}_{c} & \text { dimensionless scale of cavity } \\ L_{t} & \text { length of body } \\ \bar{L}_{t} & \text { dimensionless scale of body } \\ m_{r} & \text { reflux mass-flow-rate } \\ P_{c} & \text { transient static pressure } \\ P_{\infty} & \text { standard atmospheric pressure } \\ Q_{c} & \text { ventilated volume-flow-rate } \\ \bar{Q}_{c} & \text { ventilated coefficient }\end{array}$

$\begin{array}{cl}\bar{Q}_{r} & \text { reflux coefficient } \\ R & \text { ideal gas constant } \\ R e & \text { Reynold numbers } \\ T & \text { time variable } \\ T_{v} & \text { temperature of air } \\ U & \text { water flow speed } \\ x & \text { x-axis coordinates } \\ \beta & \text { incline angle of vortex tube } \\ & \quad \text { separation line } \\ \gamma & \text { ratio of specific air heats } \\ \sigma_{c} & \text { supercavity number } \\ \mu_{l} & \text { dynamic viscosity of water } \\ \rho_{g} & \text { density of gas }\end{array}$




$$
\begin{array}{ll}
Q_{j} & \text { jet volume-flow-rate } \\
\bar{Q}_{j} & \text { jet coefficient }
\end{array}
$$

\section{INTRODUCTION}

As a promising method to reduce the resistance of underwater vehicles, supercavity technology has been highly valued by scholars all over the world. There are two ways to achieve supercavity: natural supercavity and ventilated supercavity. The production of natural supercavity usually requires underwater objects moving at very high speeds (Karn et al. 2016; Kirschner et al. 2001; YI et al. 2008). In addition, ventilated supercavity is method commonly used in many practical applications, including high-speed torpedo (Ahn et al. 2015; Owis and Nayfeh 2002), projectile (Cameron et al. 2011; Jin et al. 2011) and marine systems (Pearce and Brandner 2012; Xu et al. 2018). It injected noncondensable gas from the nose of underwater objects in order to promote the formation of supercavity. The characteristics of the cavitator, ventilation condition and vaporous effect were noticed for understanding the formation and entrainment conditions of the different supercavities. The effect of cavitator structure and shape forming and sustaining ventilated supercavity was investigated (Balakrishna et al. 2019). In the research, three cavitators of different shapes (i.e. 2D cavitators including triangle and disk shape, and 3D coneshaped cavitator) with the same frontal area were employed to develop ventilated supercavity. The cone-shaped cavitator required the least amount of ventilation to generate a supercavity among all different shapes. A supercavity can also be formed through both ventilation and vaporization approach. Jihua (2011) observed the characteristics of ventilated supercavity formation and collapse in the NWPU high-speed water tunnel. They indicated that there was a hysteresis effect in the collapse process of supercavity generation. Karn and Chawdhary (2018) studied the synergistic relationship between the process of supercavity formation under these two modes. The results showed supercavity formation was not hysteretic because of continuous supply of air and vapor to the interface. Jiang et al. (2017) carried out a systematic experimental investigation on the concept of using a gas nozzle at the tip of the object as a ventilated hole to form supercavity. In this formation way, the supercavity was constituted by injecting gas straight ahead without cavitator. It was found that an ellipsoidal bubble located in a certain distance of the test body tip was appeared during the development of supercavity, and the ellipsoidal bubble gradually grew to a large cavity with increasing water flow velocity.

The flow characteristics of ventilated supercavity is also an important aspect on cavity formation and the stability features. Therefore, many scholars have performed numerical simulation analysis on the flow structure of the supercavity. For example, the pressure characteristic of cavity is an important

\author{
$\rho_{l} \quad$ density of liquid \\ $\Delta t \quad$ time interval
}

physical quantity, which is only measured at a designated location in the experiment. The occurrence of pressure gradients inside the cavity was often ignored (Kawakami and Arndt 2011; Lee et al. 2013). Thus, only numerical simulation methods can be used to recognize the gradient changes in the internal pressure of the cavity. The work of Rashidi et al. (2014) had shown that the pressure distribution in the entire supercavity appeared uniform. Kinzel et al. (2015) reported the distribution of pressure for different value of ventilation. Besides, other flow characteristics had also been studied through numerical simulation methods. Wang et al. (2021) established a computational model to investigate the effects of a periodic gust flow on the wake structure of ventilated supercavity. The conclusions demonstrated that the behavior of ventilated supercavity against unsteady incoming flow was more stable than the vapor cavity. Kinzel's research (Kinzel et al. 2017) also showed that the axial flow rate increased under large jet flow rate conditions, and interfacial instability occurred in the supercavity, which caused the Efros closure mode. Wang et al. (2018) distinguished the flow patterns in the wake into two different categories: unstable structure with vortex shedding and other relatively stable structures namely with Re-entrant jet or stable supercavity.

Most of the research have been explored in detail without the coupling to propulsors. If the underwater vehicle is coupled with the propulsors, the ventilated-jet supercavity is occurred. Generally, underwater supercavity vehicles use rocket motors as the driving force to move forward, and the highspeed jet gas produced by the rocket motors exerts a certain influence on the shape of supercavity. Simultaneously, the coupling interaction of supercavity and tail jet make the supercavity problem more complicated. The theoretical calculation formula of the ventilated-jet supercavity was studied in Paryshev's (Paryshev 2006) work. They gave the judgment criterion of cavity closure mode, and the complete re-entrant jet mode was that the dimensionless momentum is less than $1 / 2$. Paryshev's theoretical formula also had a good reference value for the calculation of ventilated-jet with propulsors. Additionally, in all these supercavity flow, different length bodies inside the supercavity interfered with the internal pressure. Particularly, under the critical case of supercavity collapse, the influence of body scale on supercavity is also very obviously.

In the present paper, in order to take full advantage of the exhaust gas generated by the rocket engine and solve the problem of insufficient gas source for underwater vehicles, the mechanism to sustain the main cavity by using the tail jet should be carefully investigated. A new scheme of supercavity generation which is mainly supported by the tail jet is proposed and called jet-reflux 
supercavity. The formation conditions and the entrainment mechanism of jet-reflux supercavity will be analyzed in detail. Then, the different tail closures of the ventilated supercavity are reported and a closure mechanism is selected to study the tail jet. The numerical models are constructed in accordance with the ventilated-jet supercavity experiments conducted at hydrodynamics laboratory and are validated using the experimental data. Then, by using experimental bodies of different scales as the research objects, the interaction between ventilated supercavity and tail jets is carried out to analyze the evolution process of supercavity morphology. The formation conditions of jet-reflux supercavity and the critical jet coefficient for supercavity collapse are obtained. Finally, the verified numerical method is devoted to study the internal flow of the cavity under different jet conditions, body lengths and individual ventilated conditions. This paper is structured as follows: Sec. 2 provides the details of the experimental and numerical methods employed in the current study. The validation of the numerical method using experimental data is described and we present the numerical results on the internal pressure distribution and streamlines under different supercavity conditions in Sec. 3. Subsequently, the corresponding conclusions are given in Sec. 4.

\section{METHOD}

\subsection{Experiment Method}

The experiments are performed in the gravity water tunnel (Fig. 1) to observe the shapes of ventilated supercavity and jet supercavity under the conditions of different jet coefficients. The operation of the water tunnel is controlled by a pneumatic butterfly valve between the water storage tank and the rectifying section. It is a circulation and opened-jet facility with a horizontal test section of $1.20 \mathrm{~m}$ in length. The open-circulation water tunnel has advantage of eliminating bubbles in the upstream water, which makes the ultra-high-speed jet supercavity morphology better to observe. The jetcavity experiment uses cavitator of forward strut in Fig. 2a. In front of the cavitator, a thin NACA0012 hydrofoil (Fig. 2b) strut is placed upstream of the cavitator to minimize the interaction between the formed cavity and the strut body. The interaction between the ventilated supercavity and the rear jet has strong unsteady characteristics. The experimental model adopts a rotating body structure, which is mainly composed of a nose cavitator, an intermediate section and a tail nozzle. Its outline and dimensions are shown in Fig. 2c. The cavitator adopts a disk type with a maximum diameter of $15 \mathrm{~mm}$, and 8 vent holes with $1 \mathrm{~mm}$ diameter are arranged on the circumference equidistantly.

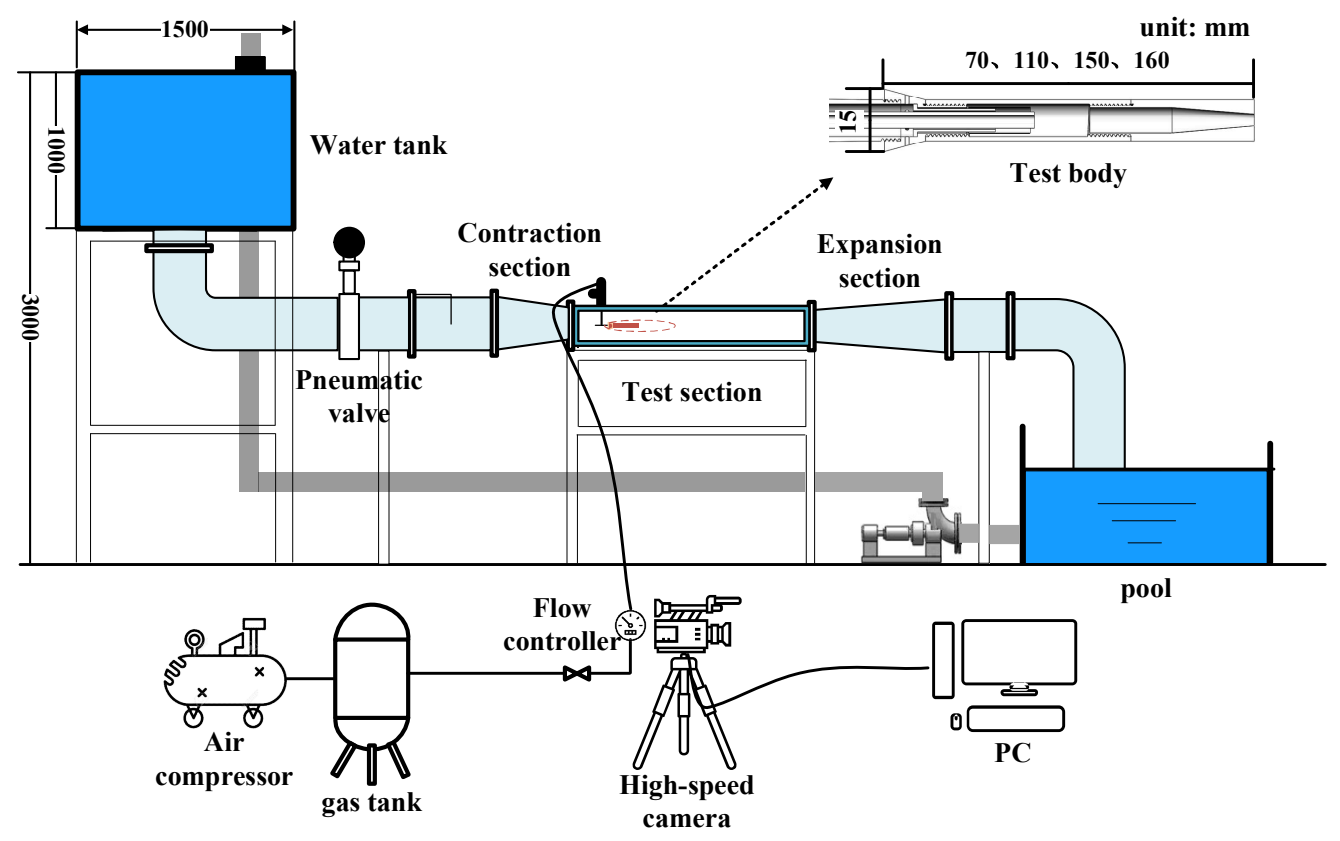

Fig. 1. Schematic of the water tunnel and its equipment at the applied hydrodynamics laboratory of the National University of Defense Technology in China.

These middle sections of different lengths can be replaced for studying the effect of relative location on the flow structure. The interior is a tail jet circulating gas pipeline. The nozzle section has a total length of $40 \mathrm{~mm}$. It is composed of a gas chamber and a shrinking nozzle.
In the experiment, the high-speed camera system uses the PCO. max S4 high-speed camera produced by the German PCO company. It has an ultra-high sensitivity, ultra-high pixel color CMOS image sensor, a full-frame resolution $(2016 \times 2016,4$ million pixels) mode. The acquisition rate is 3000 


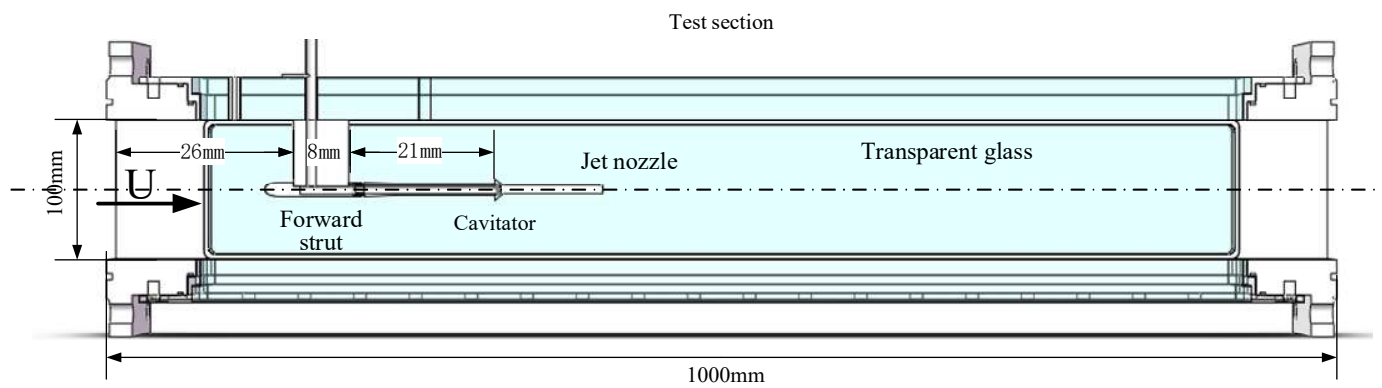

(a)

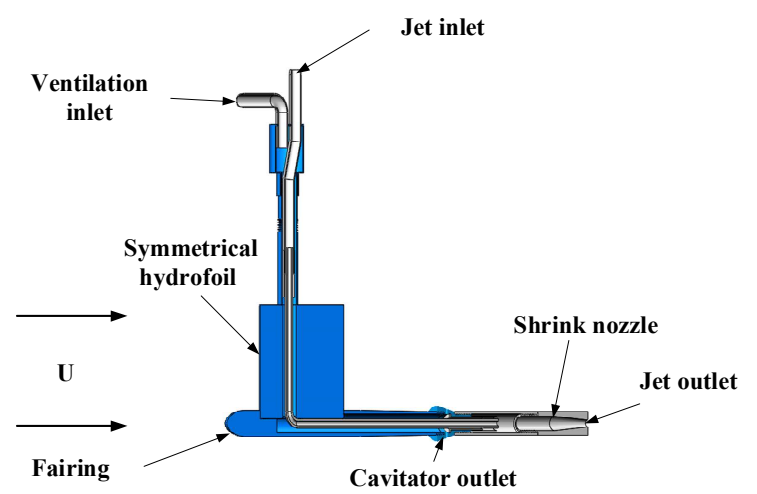

(b)

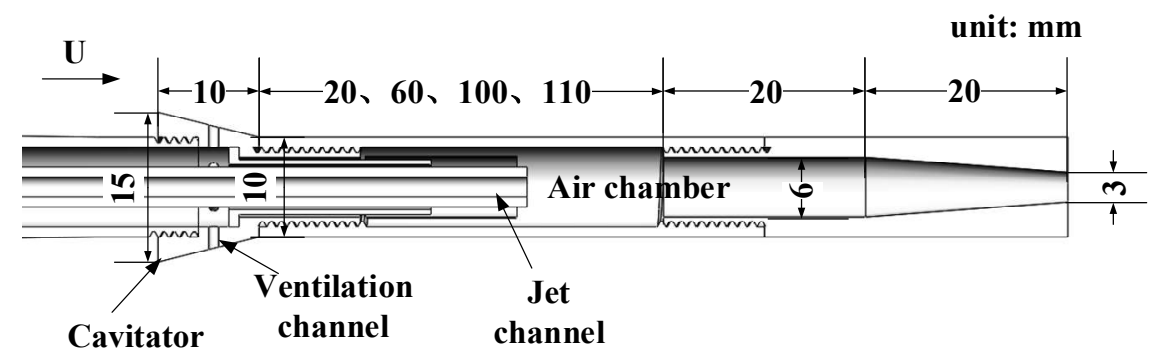

(c)

Fig. 2. (a) A side view of the test section and the mounting configuration of the backward facing cavitator, (b) A sectional view of forward strut, and (c) Schematic diagrams of channels with different body lengths.

frames/sec with the $1.5 \mu$ s shortest shutter time, and the fuselage memory is $36 \mathrm{~GB}$. It can effectively meet the needs of high-resolution, long-time and high-speed shooting. The minimum frame span time of the system is 200 milliseconds. Due to the high shooting frame rate and short exposure time, there is insufficient lighting under laboratory conditions. The light intensity can't meet the needs of highspeed photography. Under high-speed photography conditions, an additional light source is usually required to fill the measured object. The four sides of the water tunnel are all transparent plexiglass, which can be illuminated from different angles. The air flowmeters (YJ-700CD-AIR, Operation range: 010SLPM, 0-50SLPM, 0-100SLPM, 0-200SLPM, all repeatability error $<2 \%$, F.S., and response time $<1$ s) are used to control the ventilated gas and the jet flow rate.

\subsection{Numerical method}

In the experiment, the ventilated air is supplied through 8 symmetrically arranged circumferential holes at the rear of the air pocket. As shown in Fig. 3 , the variable grid size is used to maintain the fine resolution of the flow physics near the cavitator and reduce the overall computational cost. After checking the grid independence, the total number of grids is approximately $2.89 \times 10^{6}$. These holes are simplified into an endless annular band surface (Fig. 4.) in the simulation. The external flow field is simplified as the cylindrical region in order to produce a high-quality structured grid. In addition, the width of the endless annular surface has been checked to confirm its similarity to the hole. In the end structured grid is generated for the entire computational domain. 


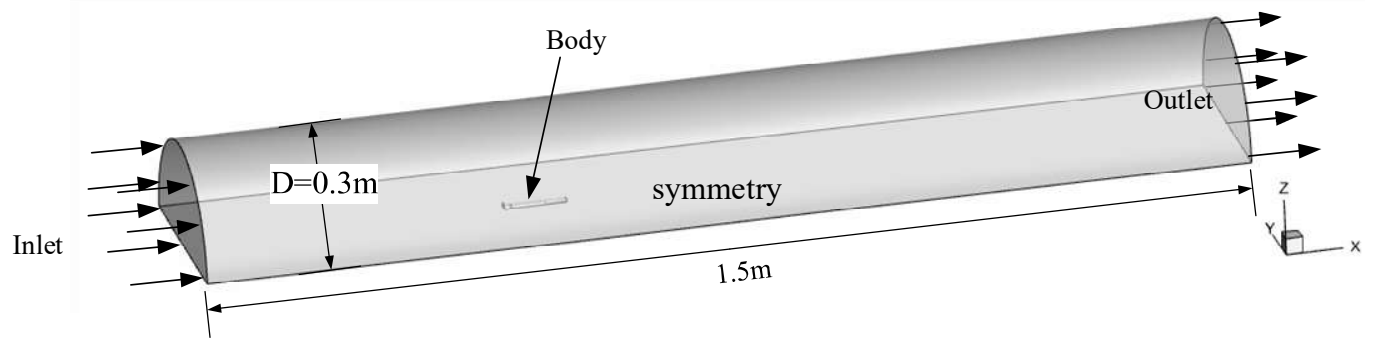

Fig. 3. Three-dimensional geometry of the computational domain (in this figure $d_{c}=15 \mathrm{~mm}$ ).

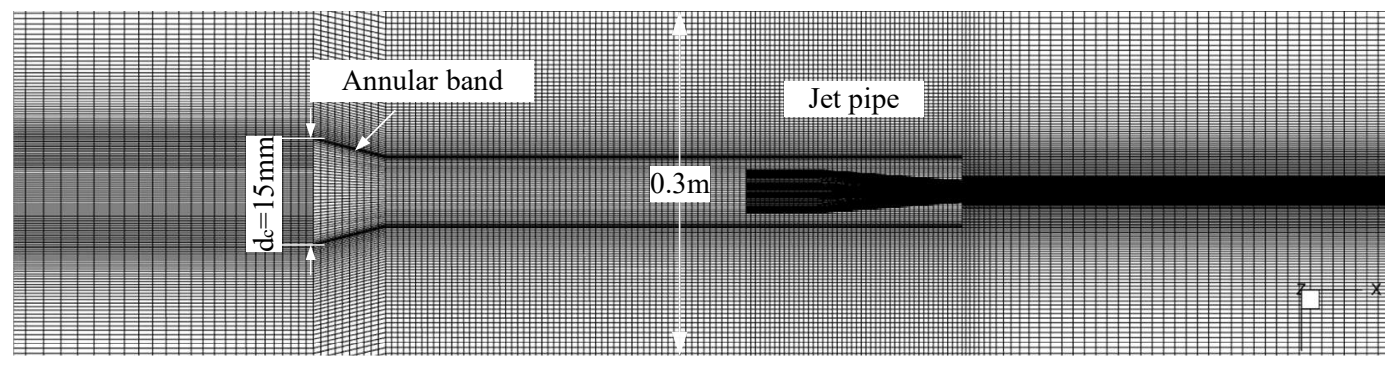

Fig. 4. Partial view of the grid on the symmetry plane.

Cavitation flow is usually considered as a vaporliquid two-phase incompressible flow. Although the two-phases do not participate in the cavitation mass transmission process for the low velocity external flow field, their existence will exert influence and constraints on the flow field and the cavitation. In addition, the generation and propagation of the pressure wave at the high-speed wake jet requires consideration of the compressibility of the flow field medium.

The governing continuity equation, momentum equation and energy equation of a homogeneous multiphase flow are given as follows:

$\frac{\partial(\rho)}{\partial t}+\nabla \square(\rho \vec{u})=0$

$\frac{\partial(\rho E)}{\partial t}+\nabla \llbracket(\vec{u}(\rho E+p))=\nabla \llbracket\left(k_{e f f} \nabla T\right)$

$$
\begin{aligned}
\frac{\partial \rho \vec{u}}{\partial t}+\nabla \cdot(\rho \vec{u} \vec{u})= & -\nabla p+\nabla \cdot \mu\left[\nabla \vec{u}+(\nabla \vec{u})^{T}\right] \\
& -\vec{F}_{s f}+\rho \vec{g}
\end{aligned}
$$

Where $\bar{u}, \rho, \mu$ represents the velocity, density, and viscosity respectively. $k_{\text {eff }}$ refers to the effective thermal conductivity shared by the phases.

Generally, the mixture density could be calculated using the phase mass fraction or phase void fraction. The maximum error may exist in the gas-liquid mixing region. The gas and liquid flow are separated by obvious interface in most regions. The phase void fraction is solved using the Volume of Fluid Method
(VOF). Therefore, the density and viscosity coefficient of the mixture phase is defined as:

$$
\begin{aligned}
& \rho=\rho_{l}\left(1-\alpha_{g}\right)+\rho_{g} \alpha_{g} \\
& \mu=\mu_{l}\left(1-\alpha_{g}\right)+\mu_{g} \alpha_{g}
\end{aligned}
$$

Where subscript $l$ and $g$ represents the liquid phase and gas phase respectively. Therefore, $\alpha_{g}$ represents the volume fraction of the air. $\alpha_{l}$ represents the volume fraction of water.

The energy $E$ is defined as mass-averaged variables:

$$
E=\frac{\alpha_{l} \rho_{l} E_{l}+\alpha_{g} \rho_{g} E_{g}}{\alpha_{l} \rho_{l}+\alpha_{g} \rho_{g}}
$$

The following state equation is adopted for liquid and gas phase:

$$
\rho_{i}= \begin{cases}p / R T, & i=g \\ \text { const } & i=l\end{cases}
$$

The gas-liquid interface is captured by solving the VOF equation:

$$
\frac{\partial\left(\rho_{g} \alpha_{g}\right)}{\partial t}+\nabla \square\left(\rho_{g} \alpha_{g} \vec{u}\right)=0
$$

To ensure mass conservation with the VOF method and keep a fine description of the geometrical properties of the interface, the level-set method is coupled to calculate the interface normal. The coupling level-set and VOF model is applied to predict the water-air two phase flows, which is a 
popular interface-tracking method for computing two-phase flows with topologically complex interfaces. In the level-set method (Osher and Sethian 1988), the interface is captured and tracked by the level-set function $\varphi$, which is defined as a signed distance from the interface. As the level-set function is smooth and continuous, the spatial gradients can be accurately calculated. This will in turn produce accurate estimates of interface curvature and surface tension force caused by the curvature. However, the level-set method is found to have a deficiency in preserving volume conservation (Olsson et al. 2007). On the other hand, the VOF method is naturally volume-conserved, as it computes and tracks the volume fraction of a particular phase in each cell rather than the interface itself. Since the VOF function (the volume fraction of a particular phase) is discontinuous across the interface, the weakness of the VOF method lies in the calculation of its spatial derivatives. The levelset function $\varphi$ is a signed distance to the interface. Accordingly, the interface is the zero level-set $\varphi(x, t)$ and can be expressed as $\Gamma=\{x \mid \varphi(x, t)=0\}$ in a two-phase flow system:

$\varphi(x, t)=\left\{\begin{array}{cl}+|d| & \mathrm{x} \in \text { the water phase } \\ 0 & \mathrm{x} \in \Gamma \\ -|d| & \mathrm{x} \in \text { the air phase }\end{array}\right.$

Where $d$ is the distance from the interface. The evolution of the level-set function can be given in a similar fashion as to the VOF model:

$$
\frac{\partial \varphi}{\partial t}+\nabla \cdot(\vec{u} \varphi)=0
$$

In Eq. (2), $\vec{F}_{s f}$ is the force arising from surface tension effects given by:

$$
\vec{F}_{s f}=\sigma \kappa \delta(\varphi) \vec{n}
$$

Where $\sigma$ is surface tension coefficient, $\kappa$ is local mean interface curvature, $\delta$ is local interface normal.

$$
\delta(\varphi)=\left\{\begin{array}{cc}
0 & |\varphi| \geq \alpha \\
\frac{1+\cos (\pi \varphi / \alpha)}{2 \alpha} & |\varphi|<\alpha
\end{array}\right.
$$

The normal $\vec{n}$ and curvature $\kappa$ of the interface can be estimated as:

$$
\begin{aligned}
& \vec{n}=\left.\frac{\nabla \varphi}{|\nabla \varphi|}\right|_{\varphi=0} \\
& \kappa=\left.\nabla \cdot \frac{\nabla \varphi}{|\nabla \varphi|}\right|_{\varphi=0}
\end{aligned}
$$

By nature of the transport equation of the level-set function Eq. (10), it is unlikely that the distance constraint of $|\nabla \varphi|=1$ is maintained after its solution.

The reasons for the lack of maintaining $|\nabla \varphi|=1$ are the deformation of the interface, uneven profile, and thickness across the interface. Those errors will accumulate during the iteration process and cause large errors in mass and momentum solutions. Therefore, a re-initialization process is required for each time step. The geometrical interface-front construction method is applied, which involves a simple concept and is reliable in producing accurate geometrical data for the interface front. The interface-front is reconstructed by the values of the VOF and the level-set function. Namely, the VOF model provides the size of the cut in the cell where the likely interface passes through, and the gradient of the level-set function determines the direction of the interface.

The ventilated-jet cavity is the three-dimensional unsteady random motion observed from moderate to high Reynolds numbers. As water-air flows are typically based on fluids of low viscosity, the wake flows are turbulent. The Reynolds Averaged NavierStokes (RANS) Turbulence Models is the most economic method for computing cavity-jet flows. And the shear-stress transport (SST) $k-\omega$ model is one of the typical RANS (Menter 2009) forms. The SST model have been designed to avoid the freestream sensitivity. In addition, the SST model has been calibrated to accurately compute flow separation from smooth surfaces. The SST $k-\omega$ model is an empirical model based on model transport equations for the turbulence kinetic energy $(k)$ and the specific dissipation rate $(\omega)$. As the $k-\omega$ model has been modified, production terms have been added to both the $k$ and $\omega$ equations, which have improved the accuracy of the model for predicting free shear flows for cavity boundary layer.

The transport equations for the SST $k-\omega$ model are given as follows:

$\frac{\partial(\rho k)}{\partial t}+\frac{\partial\left(\rho k u_{i}\right)}{\partial x_{i}}=\frac{\partial\left(\Gamma_{k} \frac{\partial k}{\partial x_{j}}\right)}{\partial x_{j}}+G_{k}-Y_{k}$

$\frac{\partial(\rho \omega)}{\partial t}+\frac{\partial\left(\rho \omega u_{i}\right)}{\partial x_{i}}=\frac{\partial\left(\Gamma_{k} \frac{\partial \omega}{\partial x_{j}}\right)}{\partial x_{j}}+G_{\omega}-Y+D_{\omega}$

Where $G_{k}$ represents the generation of turbulence kinetic energy due to velocity gradients. $G_{\omega}$ represents the generation of $\omega \cdot \Gamma_{k}$ and $\Gamma_{\omega}$ represent the effective diffusivity of $k$ and $\omega$, respectively. $Y_{k}$ and $Y_{\omega}$ represent the dissipation of $k$ and $\omega$ due to turbulence. All of the above terms are calculated as described below. $S_{k}$ and $S_{\omega}$ are source terms. $D_{\omega}$ represents the introduction of a cross-diffusion term.

The effective diffusivities for the $k-\omega$ model are given by: 
B. Liu et al. / JAFM, Vol. 15, No. 1, pp. 293-309, 2022.

$\Gamma_{k}=\mu+\frac{\mu_{t}}{\sigma_{k}}$

$\Gamma_{\omega}=\mu+\frac{\mu_{t}}{\sigma_{\omega}}$

Where $\sigma_{k}$ and $\sigma_{\omega}$ are the turbulent Prandtl numbers for $k$ and $\omega$, respectively.

$$
\begin{gathered}
\sigma_{k}=\frac{1}{F_{1} / \sigma_{k, 1}+\left(1-F_{1}\right) / \sigma_{k, 2}} \\
\sigma_{\omega}=\frac{1}{F_{1} / \sigma_{\omega, 1}+\left(1-F_{1}\right) / \sigma_{\omega, 2}}
\end{gathered}
$$

The turbulent viscosity, $\mu_{t}$, is computed by combining $k$ and $\omega$ as follows:

$$
\begin{aligned}
& \mu_{t}=\frac{\rho k}{\omega} \frac{1}{\max \left[\frac{1}{\alpha^{*}}, \frac{S F_{2}}{\alpha_{1} \omega}\right]} \\
& F_{2}=\tanh \left(\max \left[2 \frac{\sqrt{k}}{0.09 \omega y}, \frac{500 \mu}{\rho y^{2} \omega}\right]\right) \\
& D_{\omega}^{+}=\max \left[2 \rho \frac{1}{\sigma_{\omega, 2}} \frac{1}{\omega} \frac{\partial k}{\partial x_{j}} \frac{\partial \omega}{\partial x_{j}}, 10^{-10}\right] \\
& \alpha^{*}=\alpha_{\infty}^{*}\left(\frac{\frac{\beta_{i}}{3}+\frac{\rho k}{\mu \omega} / R_{k}}{1+\frac{\rho k}{\mu \omega} / R_{k}}\right) \\
& \beta_{i}=F_{1} \beta_{i, 1}+\left(1-F_{1}\right) \beta_{i, 2}
\end{aligned}
$$$$
F_{1}=\tanh \left(\min \left[\max \left(\frac{\sqrt{k}}{0.09 \omega y}, \frac{500 \mu}{\rho y^{2} \omega}\right), \frac{4 \rho k}{\sigma_{\omega, 2} D_{\omega}^{+} y^{2}}\right]\right)
$$

The coefficient $\alpha^{*}$ damps the turbulent viscosity leading to a low-Reynolds number correction. The $S$ is the strain rate magnitude and $y$ is the distance to the next surface. $D_{\omega}^{+}$is the positive portion of the cross-diffusion term. $D_{\omega}$ is defined as follow:

$D_{\omega}=2\left(1-F_{1}\right) \rho \frac{1}{\omega \sigma_{\omega, 2}} \frac{\partial k}{\partial x_{j}} \frac{\partial \omega}{\partial x_{j}}$

The term $G_{k}$ (Eq. 15) represents the production of turbulence kinetic energy. From the exact equation for the transport of $k$, this term may be defined as follow:

$$
G_{k}=-\rho \overline{u_{i}^{\prime} u_{j}^{\prime}} \frac{\partial u_{j}}{\partial x_{i}}
$$

The term $G_{\omega}$ (Eq. 16) represents the production of $\omega$ and is given by:
$G_{\omega}=\frac{\alpha \alpha^{*}}{v_{t}} G_{k}$

$\alpha=\frac{\alpha_{\infty}}{\alpha^{*}}\left(\frac{\alpha_{0}+\frac{\rho k}{\mu \omega} / R_{\omega}}{1+\frac{\rho k}{\mu \omega} / R_{\omega}}\right)$

$\alpha_{\infty}=F_{1} \alpha_{\infty, 1}+\left(1-F_{1}\right) \alpha_{\infty, 2}$

$\alpha_{\infty, 1}=\frac{\beta_{i, 1}}{\beta_{\infty}^{*}}-\frac{\kappa^{2}}{\sigma_{\omega, 1} \sqrt{\beta_{\infty}^{*}}}$

$\alpha_{\infty, 2}=\frac{\beta_{i, 2}}{\beta_{\infty}^{*}}-\frac{\kappa^{2}}{\sigma_{\omega, 2} \sqrt{\beta_{\infty}^{*}}}$

The term $Y_{k}$ (Eq. 15) represents the dissipation of turbulence kinetic energy, and is defined as follows:

$Y_{k}=\rho \beta^{*} k \omega$

$\beta^{*}=\beta_{i}^{*}\left[1+\zeta^{*} F\left[M_{t}\right]\right]$

$\beta_{i}^{*}=\beta_{\infty}^{*}\left(\frac{4 / 15+\left(\frac{\rho k}{\mu \omega} / R_{\beta}\right)^{4}}{1+\left(\frac{\rho k}{\mu \omega} / R_{\beta}\right)^{4}}\right)$

The compressibility function $F\left[M_{t}\right]$ is given by:

$$
\begin{aligned}
& F\left[M_{t}\right]=\left\{\begin{array}{cc}
0 & M_{t} \leq M_{t 0} \\
M_{t}^{2}-M_{t 0}^{2} & M_{t} \geq M_{t 0}
\end{array}\right. \\
& M_{t}^{2}=\frac{2 k}{a^{2}} \\
& a=\sqrt{\gamma R T_{v}}
\end{aligned}
$$

The term $Y_{\omega}$ (Eq. 16) represents the dissipation of $\omega$, and is defined as follows:

$Y_{\omega}=\rho \beta \omega^{2}$

$\beta=\beta_{i}\left[1-\frac{\beta_{i}^{*}}{\beta_{i}} \zeta^{*} F\left(M_{t}\right)\right]$

$\beta_{i}=F_{1} \beta_{i, 1}+\left(1-F_{1}\right) \beta_{1,2}$

The shear-stress transport (SST) $k-\omega$ model constants:

$$
\begin{aligned}
& \sigma_{k, 1}=1.176, \sigma_{\omega, 1}=2.0, \sigma_{k, 2}=1.0, \sigma_{\omega, 2}=1.168 \\
& \alpha_{1}=0.31, \beta_{i, 1}=0.072, \beta_{i, 2}=0.0828, \kappa=0.41 \\
& \alpha_{\infty}^{*}=1, R_{k}=6, R_{\omega}=2.95, R_{\beta}=8, M_{t 0}=0.25 \\
& \zeta^{*}=1.5, \beta_{\infty}^{*}=0.09
\end{aligned}
$$

The SST $k-\omega$ turbulence model is compared with the standard $k-\varepsilon$ turbulence model, which has shown superiority for the shedding period and gasliquid interface prediction (Menter 1994). The SST 
$k-\omega$ turbulence model had shown superiority for separated flow and high shear flows (Menter 1992) for this research. Therefore, the SST $k-\omega$ model does produce a bit high precision in regions with large normal strain, like wake regions of cavity and regions with strong gas acceleration.

\subsection{Validation of Numerical Model}

Firstly, numerical simulation is firstly carried out for the ventilated supercavity with tail jet flow. The Froude number is set as 13.4 and the jet coefficient is $\bar{Q}_{j}=4$. The corresponding Reynolds number is about 45000 for both experiments and simulations. The gas-liquid interface is compared with the corresponding experimental situation to verify the numerical method shown in Fig. 5. Under the action of the tail jet flow, the ventilated supercavity worked at the continuous shedding mode. The captured cavity profile and the closure position match well with the experimental data. The characteristic length $\left(L_{c}\right)$ is defined as the distance from the cavitator to the closed position of the cavity and is in consistent with the experimental picture. However, the maximum diameter is underpredicted due to the ignoration of the forward struct in the simulation. In general, this comparison validates the numerical method and provides a basis for further study of the detail flow structure. The result of the numerical prediction is compared with the experimental data in Fig. 6. The maximum error between the predicted cavity length and the experimental data was within $1 \%$. Because the effects of the forward strut are not considered in the numerical model, the between predicting maximum of cavity diameter and experimental data is around $14.8 \%$ error as shown in the Fig. 6.

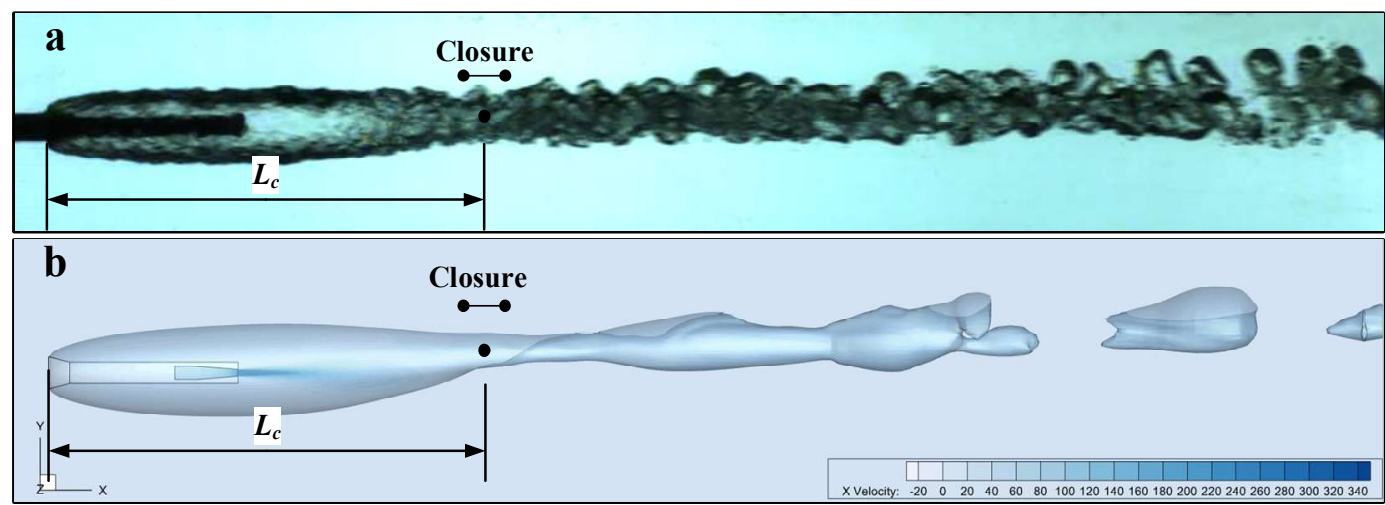

Fig. 5. Comparison of experimental and numerical results (a) photograph of jet-reflux supercavity from experiment; (b) The numerical distribution of the iso-surface with an air volume fraction of 0.5 on the symmetry plane.

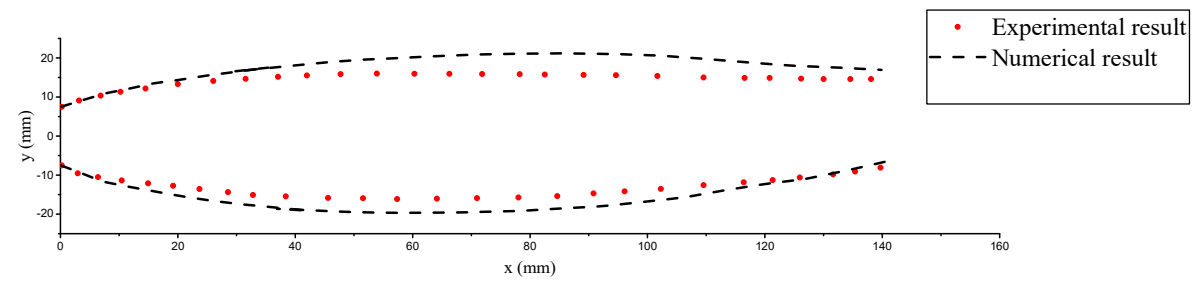

Fig. 6. Error analysis of boundary outline in experiment and numerical calculation for jet-reflux cavity.

\section{RESULT AND DISCUSSION}

\subsection{Research on the Formation Mechanism of Supercavity by Jet Reflux of Rear}

In this research, the interaction mechanism between the ventilated supercavity and tail jet around the body with a backward configuration is investigated experimentally. Two types of ventilated supercavity are observed in research: one refers to the traditional supercavity generated by the ventilating gas. While the other refers to the supercavity formed by the tail jet reflux. The above supercavity is called as jet- reflux supercavity. It is a mode that rear jet gas flows to the cavitator, which will be specifically explained in the following subsections. The important dimensionless parameters which affect the cavity characteristics and pressure distribution are ventilated coefficient $\left(\bar{Q}_{c}\right)$, jet coefficient $\left(\bar{Q}_{j}\right)$ and supercavity number $\sigma_{c}$ etc. The corresponding standard ventilated coefficients $\left(\bar{Q}_{c}\right)$ and jet coefficient $\left(\bar{Q}_{j}\right)$ are similar to Karn et al. in research. (Karn et al. 2016). The ventilated coefficient is defined as: 


$$
\bar{Q}_{c}=Q_{c} / U d_{c}^{2}
$$

Where $Q_{c}$ is the volume-flow-rate in standard liters per minute of artificial ventilation, $U$ is define as water speed and $d_{c}$ is the diameter of the cavitator. The jet coefficient is defined as:

$\bar{Q}_{j}=Q_{j} / U d^{2}$

Where $Q_{j}$ is the volume-flow-rate in standard liters per minute of the tail jet, $U$ is define as water speed and $d$ is the diameter of the body.

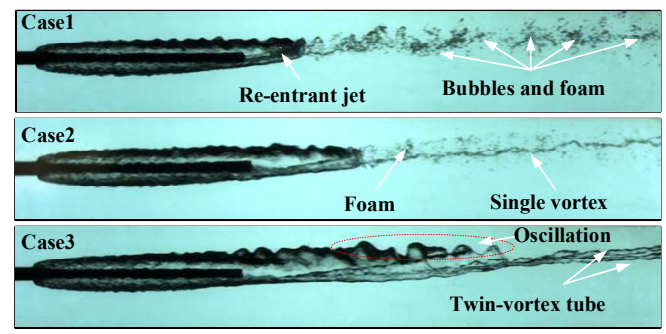

Fig. 7. Some regimes of ventilated supercavity closure: case1), Re-entrant jet off body; case2), single-vortex tube off body; case3), Twin-vortex tube off body.

For the supercavity without tail jet flow, three closure regimes are observed as shown in Fig. 7: case1), Re-entrant jet closure; case2), single-vortex closure, case3), twin-vortex closure. The re-entrant jet closure is formed when the ventilated coefficient is less than 0.03 . Numbers of bubbles exit from the closed position and then disperse into tiny foam. As the ventilated coefficient $\left(\bar{Q}_{c}\right)$ is raised to 0.04 , a single vortex closure pattern (Fig. 7 case2) is observed. This has never been mentioned in the other research (Karn et al. 2016). The single-vortex closure occurs at the transition stage between twinvortex closure and re-entrant jet closure and is characterized by a foamy and stable vortex tube. When the ventilated coefficient is over 0.06 , the downstream of the supercavity obviously shows pair of vortex tube, which is commonly observed in the other experiments.

In the subsequent experimental studies, the interaction between ventilation and jet will be analyzed on the basis of re-entrant jet closure. During the jet-reflux experiment, the ventilated channel and the tail jet channel are controlled in time-sequence. As shown in Fig. 8, the ventilated channel in nose is triggered at time $T_{0}$. After passing $\Delta T$ period, the ventilated supercavity is formed to cover the whole body; Subsequently the tail jet channel is opened at time $T_{1}$. After $\Delta T$ period, the supercavity is continuously shrinking under the action of the high-speed tail jet flow. Then, the ventilated channel is closed at time $T_{2}$, which means that only the tail jet flow is still working. A periodically stable pattern is finally achieved at time
$T_{2}+\Delta t$. Fig. 8 also presents the evolution of the supercavity surface with different jet coefficient. Two flow patterns are observed at time $T_{2}+\Delta t$. With lower jet coefficient shown in Fig. 8a, the supercavity is still maintained by the jet reflowing when the ventilated channel in nose is closed. With high jet coefficient shown in Fig. 8b, the supercavity breaks when the nose ventilation is shut down. The flow structure switches to the traditional submerged jet flow mode (Xiang et al. 2021) that a periodically expansion, necking and breakage process occur at downstream of the jet outlet. It can be induced that part of the tail jet can be directed forward to sustain the supercavity under some conditions, which may replace the nose ventilated system. When the jet flow is improved, the supercavity will be destructed. In order to utilize the tail jet flow effectively, it is critical to reveal the formation mechanism of the jetreflux supercavity. Therefore, combining with the influences between jet coefficient and the length of the bodies, the collapse process of the jet-reflux supercavity is study in Fig. 9. The different cases of the bodies are listed in Table 1. For jet-reflux supercavity, there are two modes of gas leakage. The first one is the coexistence of the two-vortex tube and oscillation as shown in the cases 1 3 of Fig. 9a, and the details are described in case 3 . The other one is the jet leakage mode that the bubbles periodically bulge and pinch off, as shown in the latter cases in Fig. 9a. The experimental results show that the length of cavity decreases as the jet coefficient ( $\bar{Q}_{j}$ ) increases in Fig. 9b. The above results are caused by the pressure change inside the cavity. Under the condition of high-speed jet, the local static pressure of the rear inside cavity becomes lower, and the pressure near the external water flow interface is higher than the internal pressure, which produces a compression effect on the cavity. As the jet coefficient increases, the two-vortex tube does not

Table 1 Experimental data of different cases.

\begin{tabular}{|c|c|c|c|c|c|c|}
\hline \multirow{4}{*}{ Type } & Case & $\begin{array}{c}L_{t}, \\
\mathrm{~mm}\end{array}$ & $\begin{array}{c}Q_{c}, \\
\mathrm{SLP} \\
\mathrm{M}\end{array}$ & $\bar{Q}_{c}$ & $\begin{array}{c}Q_{j}, \\
\mathrm{SLP} \\
\mathrm{M}\end{array}$ & $\bar{Q}_{j}$ \\
\hline \multirow{4}{*}{$\begin{array}{c}\text { Ventilated } \\
\text { cavity }\end{array}$} & 1 & 160 & 2.5 & 0.03 & 0 & 0.00 \\
\cline { 2 - 7 } & 2 & 160 & 3 & 0.04 & 0 & 0.00 \\
\cline { 2 - 7 } & 3 & 160 & 5 & 0.06 & 0 & 0.00 \\
\hline \multirow{5}{*}{ Jet-reflux } & 4 & 70 & 0 & 0 & 30 & 0.99 \\
\cline { 2 - 8 } cavity & 5 & 70 & 0 & 0 & 50 & 1.67 \\
\cline { 2 - 8 } & 6 & 70 & 0 & 0 & 60 & 2.00 \\
\cline { 2 - 8 } & 8 & 70 & 0 & 0 & 70 & 2.33 \\
\cline { 2 - 8 } & 9 & 70 & 0 & 0 & 90 & 3.00 \\
\cline { 2 - 7 } & 10 & 70 & 0 & 0 & 100 & 3.33 \\
\cline { 2 - 7 } & 11 & 70 & 0 & 0 & 120 & 4.00 \\
\hline
\end{tabular}


B. Liu et al. / JAFM, Vol. 15, No. 1, pp. 293-309, 2022.

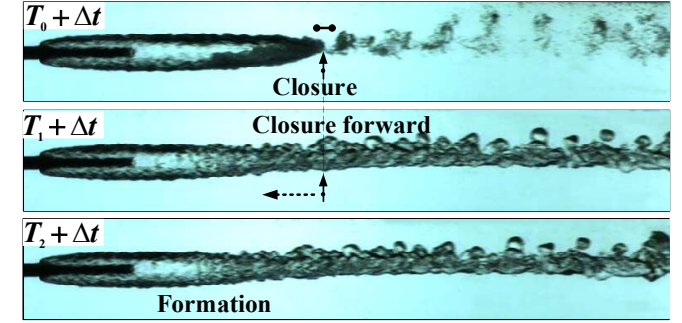

(a)The supercavity can be maintained morphology.

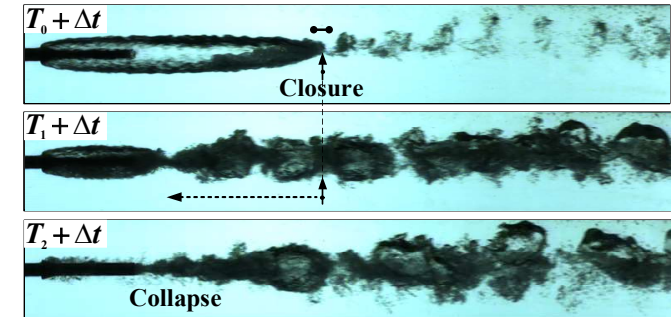

(b)The supercavity collapse flows morphology.

Fig. 8. Flow pattern diagram of supercavity morphology change: (a) and (b).

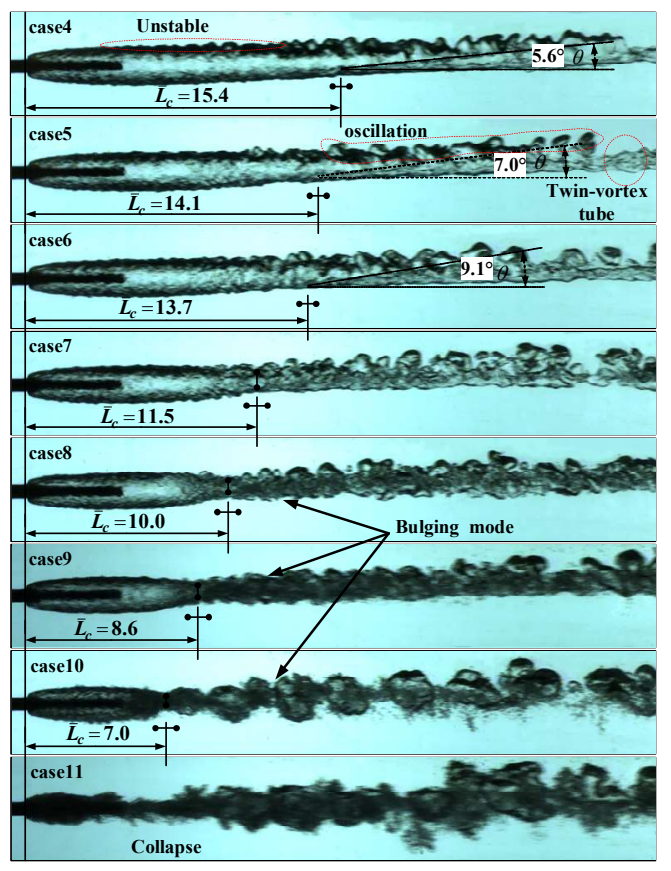

(a) Body 1

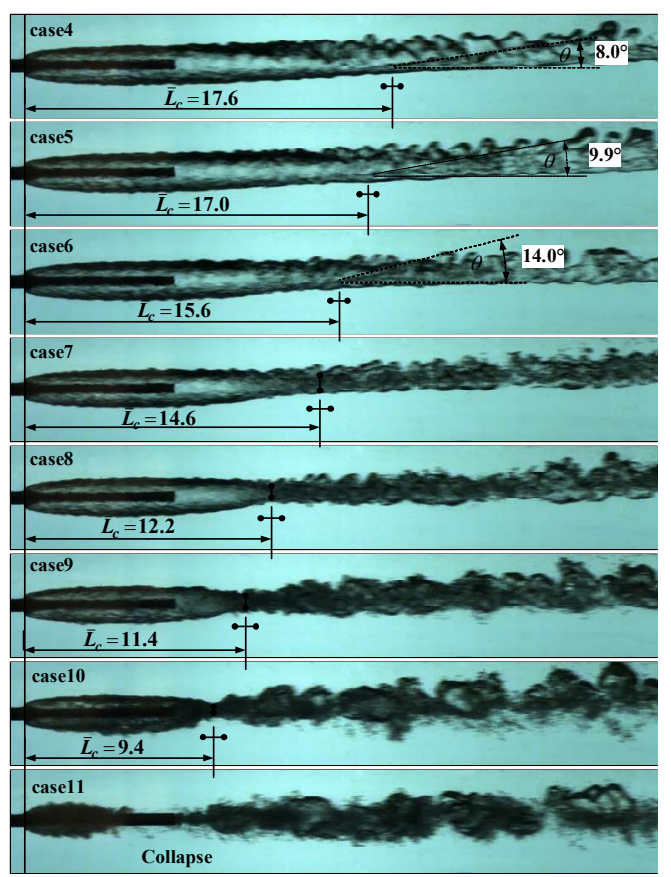

(b) Body 2

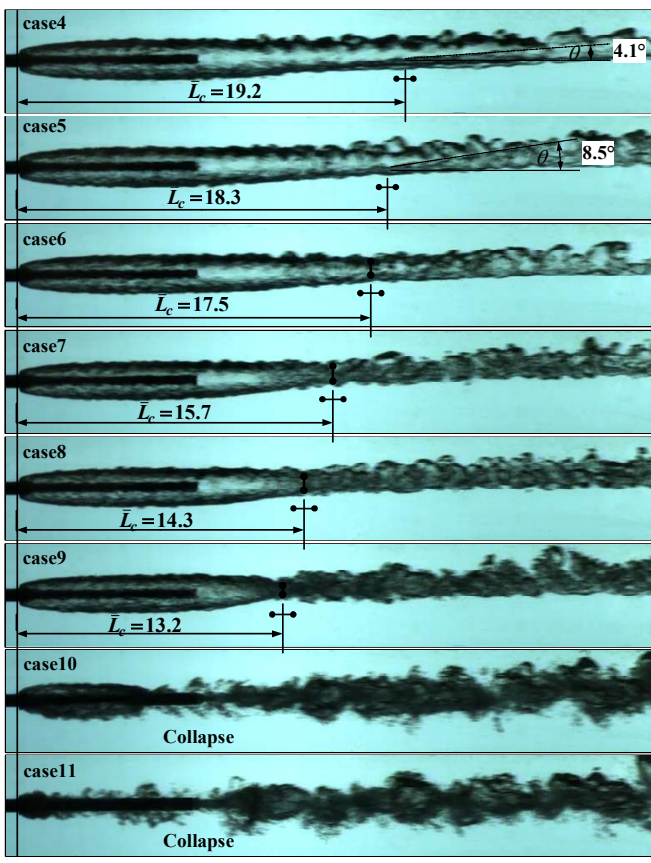

(c) Body3

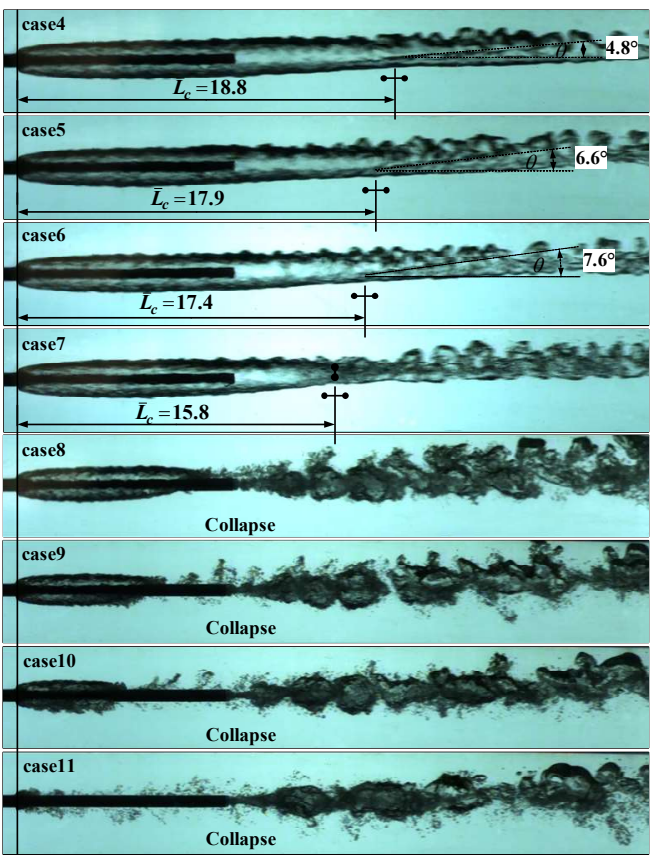

(d) Body4

Fig. 9. Collapse process of supercavity by the reflux of the rear gas at different $\bar{L}_{t}$ numbers.

(a) $\bar{L}_{t}=4.67$, (b) $\bar{L}_{t}=7.34$, (c) $\bar{L}_{t}=8.67$, (d) $\bar{L}_{t}=10.67$. 
become wider, but the oscillating bubble presents a larger volume and higher shedding speed to allow more gas leak from the cavity in Fig. 9a. As a result, mass conservation is satisfied for the jet flow input and gas leaking. Additionally, the upper water-gas interface is extremely unstable (Fig. 9a) for oscillation and two-vortex tube leakage. For this coupling leakage mode, the incline angle of twovortex tube separation line $(\beta)$ is defined in Fig. 9, which will enlarge with an increased jet coefficient. In this stage, the pressure inside the supercavity is less than the ambient liquid pressure.

The faster local gas velocity leads to a lower local pressure. Under the action of buoyancy, the angle of the separation line of the two-vortex tube increases to satisfy the force balance. Therefore, the shorter length of the cavity is cause by the effect of buoyancy, which leads to the greater value of the separation angle for the twin-vortex tube. When the jet coefficient reaches a certain value, the coupling leakage mode converts the periodic expansion and pinch off mode. It is similar to the shedding scheme of the submerged jet flow. In this leakage mode, the tail of the supercavity would bulge, shrink and then be pinched off. With higher jet coefficient, a large number of liquid droplets are rolled into the separated bubbles, in which leads to a darker image of cases $8 \sim 11$ in Fig. 9a. Meanwhile the effect of buoyancy is gradually weakened, so that the main cavity does not float upwards and then becomes an axisymmetric shape completely. When the jet strength becomes strong enough, the position of the closure moves towards the nozzle outlet. And a strong reflux is observed inside the cavity (Fig. 12), which leads to the fluctuation of the cavity surface. Finally, the jet-reflux supercavity is collapsed and the flow presents as a pure submerged jet mode of cases $10 \sim 11$ in Fig. 9c. Comparing the test bodies with different scales, the supercavity maintains stable and transparent state with low jet coefficient of cases 4 6 in Fig. 9a. Yet, as the increasing length of the test body, the length of cavity is extended with the same jet of case 7 in Fig. 9.

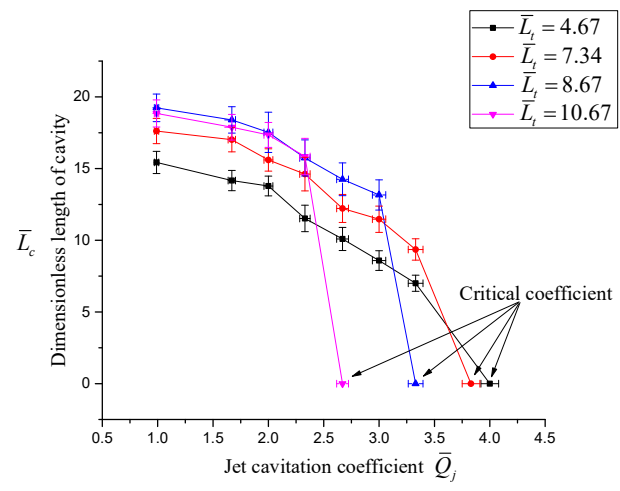

Fig. 10. Curve of scale changing with jet-reflux coefficient for various bodies.

For the leakage mode of two-vortex tube and oscillation surface, the position of the closure is the starting position of the twin-vortex tube, which is a kind of steady flow state and the starting position moves unconspicuously. The data point in each diagram is obtained from at least three tests and $\pm 2 \%$ repeatability error bars are included for the position of the closure. For the leakage mode of the bubbles bulge and pinch off, the closure position, which is also called the necking position, is located at the minimum diameter of cavity rear. However, the necking position often moves forward or backward duo to the tail jet flow. Therefore, the cavity length is also measured from the pictures obtained from three identical tests, and $\pm 5 \%$ repeatability error bars are included for the closure position. The dimensionless scale of cavity is defined as:

$\bar{L}_{c}=\frac{L_{c}}{d_{c}}$

Where $L_{c}$ is the length of cavity and $d_{c}$ is the diameter of the cavitator. The dimensionless scale of body is defined as:

$\bar{L}_{t}=\frac{L_{t}}{d_{c}}$

Where $L_{t}$ is the length of cavity and $d_{c}$ is the diameter of the cavitator.

According to the jet coefficient of different test bodies, the change curve of the cavity length $\left(\bar{L}_{c}\right)$ is shown in Fig. 10. The dimensionless conversion of $\bar{L}_{c}, \bar{L}_{t}$ and $\bar{Q}_{j}$ are implemented. It can be seen that the length of cavity decreases with the jet coefficient. Under the same jet coefficient, the dimensionless scale of cavity extends with the increasing length of test body. However, when the $\bar{L}_{t}$ number improve to a certain value, the scale of cavity reaches the maximum size limit. As shown in Fig. 10, the maximum length of $\bar{L}_{t}=10.67$ is equal to curve $\bar{L}_{t}=8.67$. The critical jet coefficient of collapse diminishes with the extension of the body length. It can be concluded that the jet outlet is closer to the cavitator, it is more conducive to maintain the jet-reflux supercavity. However, when the highspeed jet is too close to the cavitator, the pressure vibration will be transferred to the head region, which leads to fluctuation of the cavity interface in case 4 of Fig. 9a. This will be detrimental to the jetreflux supercavity.

\subsection{Analysis of Entrainment Characteristics based on Numerical Simulation}

\subsubsection{Detail flow structure analysis}

In order to investigate on the formation of the jetreflux supercavity, the detail flow structure of the supercavity will be discussed based on the numerical results. Fig. 11 shows the pressure distribution inside the ventilation supercavity and the jet-reflux supercavity respectively. From the different pressure distributions mentioned above, it can be concluded that a larger pressure gradient is observed inside the jet-reflux supercavity, and the location of pressure 
gradient is in the middle of nozzle outlet and closed position of cavity. For the ventilated supercavity, the pressure is almost uniformly distributed inside the supercavity, while a great pressure gradient is observed at the closure position. With the similar supercavity size, the jet coefficient is much higher

than the ventilated coefficient. This is attributed to the change of the shedding scheme from the reentrant jet to the bulging shedding scheme. The steep pressure gradient inside the jet-reflux supercavity occurs around the jet shear layer and upstream of the closure position. The large momentum of the tail jet can result in high velocity gradient and low pressure near the jet-reflux region. These pressure distributions are related to the flow structures inside the supercavity in Fig. 12. Comparing with the ventilated supercavity, the length-to-diameter ratio is increased with the jet and the cavity shape is less affected by buoyancy force. The pressure variation inside the supercavity is in accordance with the change of velocity, which can be presented by the streamline characteristics. The following analysis will focus on the streamline feature to analyze how the gas inside the cavity moves.

Fig. 12 shows the streamline characteristics of ventilated supercavity and jet-reflux supercavity. It is found that another large recirculation region is formed inside the jet-reflux supercavity. The gas of the tail is ejected to form a maximum recirculation zone above the $\mathrm{x}$-axis, which extends from the nozzle outlet to the necking location, while another counter rotating vortex is formed below the $\mathrm{x}$-axis.

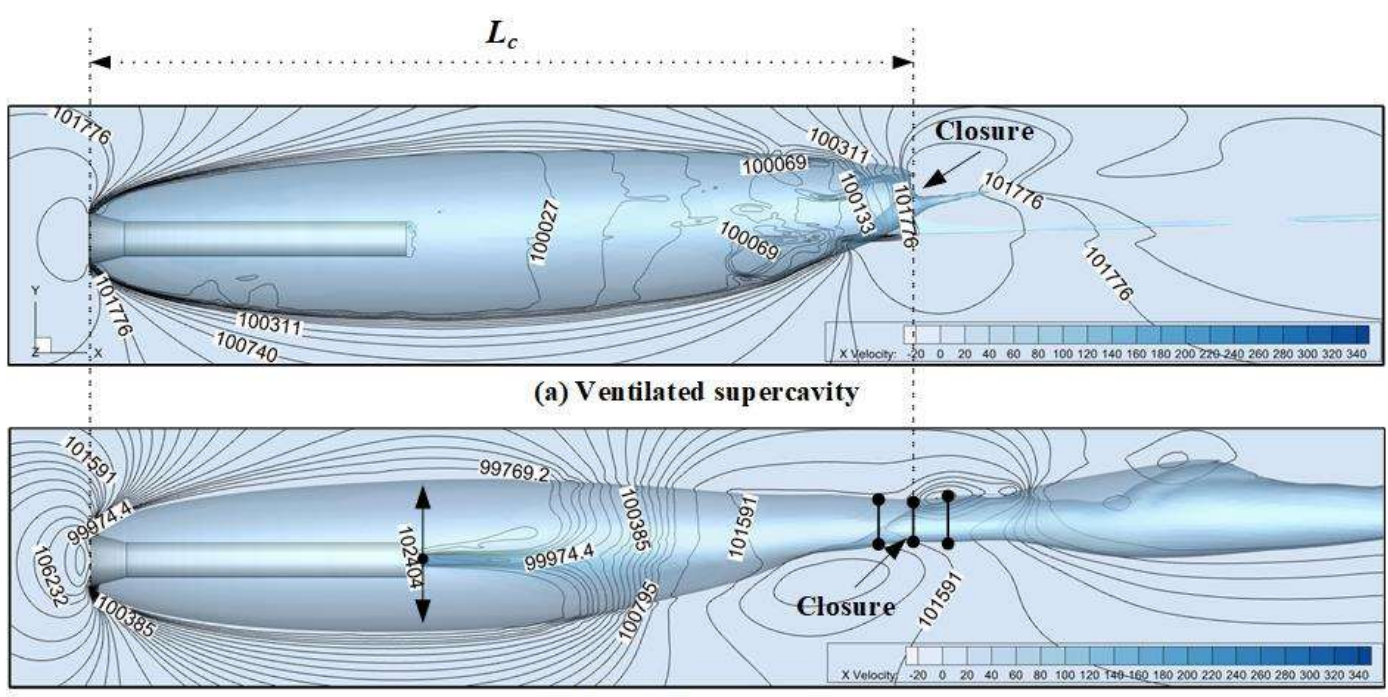

(b) Jet-reflux supercavity

Fig. 11. Static pressure distribution on the symmetry plane with different supercavities.

(a) $\bar{Q}_{c}=0.77, \bar{Q}_{j}=0$, (b) $\bar{Q}_{c}=0, \bar{Q}_{j}=3.61$.

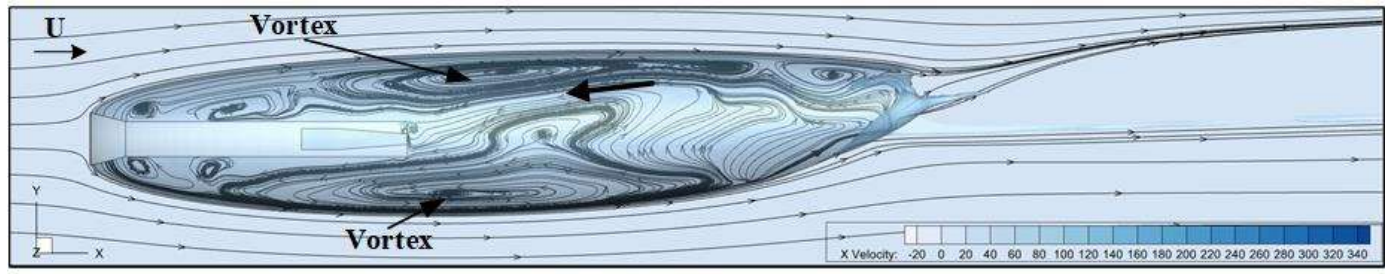

(a)Ventilated supercavity

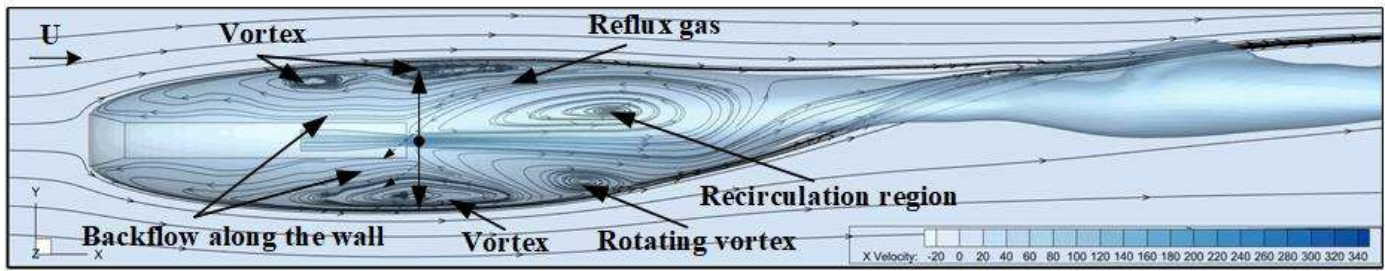

(b) Jet-reflux supercavity

Fig. 12. 2D streamline description on the symmetry plane of the same supercavity scale for (a) ventilated supercavity $\bar{Q}_{c}=0.77, \bar{Q}_{j}=0$, and (b) jet-reflux supercavity $\bar{Q}_{c}=0, \bar{Q}_{j}=3.61$. 


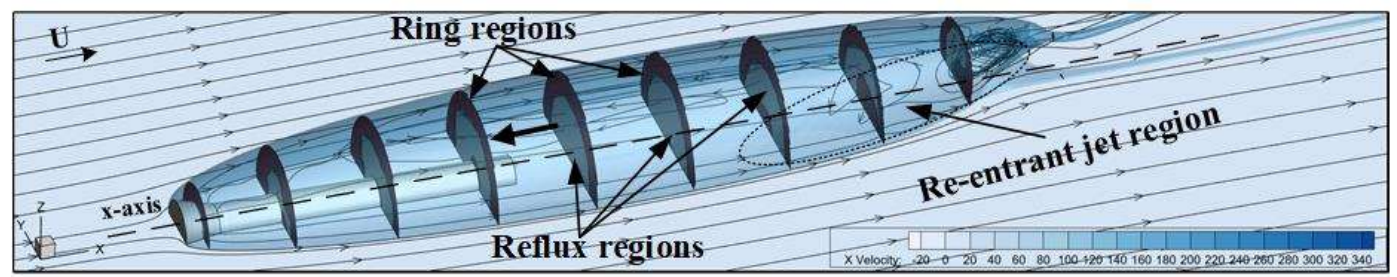

(a) Ventilated supercavity

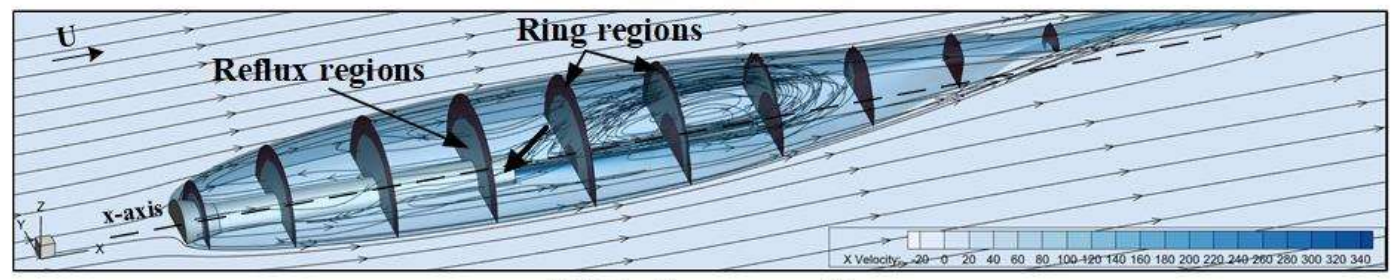

(b) Jet-reflux supercavity

Fig. 13. 3D streamlined display of the gas reflux inside the supercavity for (a) ventilated supercavity $\bar{Q}_{c}=0.77, \bar{Q}_{j}=0$, and (b) jet-reflux supercavity $\bar{Q}_{c}=0, \bar{Q}_{j}=3.61$.

Then a part of the reflux gas flows towards the cavitator and turns at the head to flow downstream along the cavity boundary layer. The others recirculated inside the induced vortex regions. As shown in Fig. 13, the path of ventilated gas and jet gas are presented by the three-dimensional streamline distributions. The xy-symmetric plane is rotated $120^{\circ}$ around the $\mathrm{x}$-axis, and then rotated $60^{\circ}$ around the z-axis to clearly observe the threedimensional flow characteristics. The gas downstream flows from the head along the cavity boundary layer which is represented by the ring regions displayed with black color in the vertical planes. Similarly, the gas flowing back through the central reflux regions is displayed in blue color. The gas inside the ventilated supercavity backflows along the $\mathrm{x}$-axis except re-entrant jet region. As for the jet-reflux supercavity, the gas backflows from the maximum recirculation region, and then downwards flows crossing the xysymmetric plane to provide gas for the lower side of the cavity. Therefore, the jet-reflux supercavity presents obvious three-dimensional inflating flow characteristics, which is different from the axisymmetric mode of the ventilated supercavity.

The formation of the jet-reflux supercavity depends on the reflux rate. The number of reflux rate affects the scale and growth law of cavity to a certain degree. Therefore, it is particularly important to investigate on the reflux rate under different conditions. Fig. 14 shows the variation of the reflux coefficients (Eq. 47) of two kinds of supercavity along the $\mathrm{x}$-axial position. The reflux coefficient is the measure of the gas backflow rate through different vertical section-region, which reflects the mass of the backflow gas inside the supercavity. Multiple vertical planes are established along $\mathrm{x}$-axial of the supercavity as shown in Fig. 14, and the mass flow of air passing through each reflux region is obtained by weighted average $\mathrm{x}$-velocity.

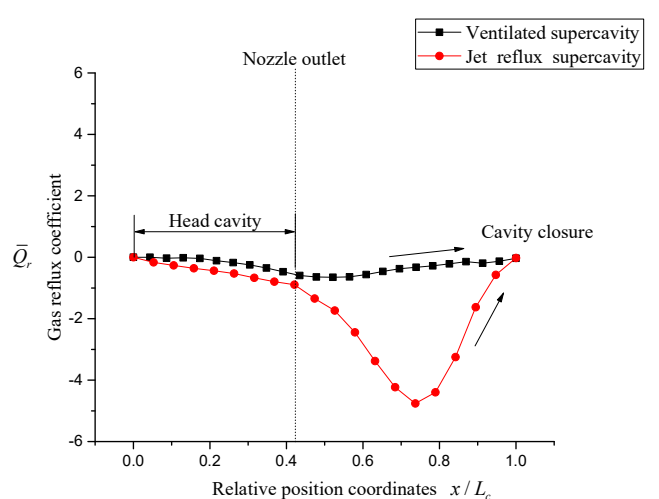

Fig. 14 Variation of reflux coefficient corresponds to the position of the supercavity.

As the result of the downstream mass-flow-rate ( $\left.m_{r}>0\right)$ and the reflux mass-flow-rate $\left(m_{r}<0\right)$, the velocity is essential decomposed into two directions along the $\mathrm{x}$-axis. However, it is very concerned about the effect of the reflux mass-flow-rate $\left(m_{r}<0\right)$ on the supercavity. Thus, the reflux mass-flow-rate is converted to dimensionless gas reflux coefficient $\bar{Q}_{r}$ :

$\bar{Q}_{r}=\frac{m_{r}}{\rho_{g} U d_{c}^{2}}$

Where $m_{r}$ is defined as the reflux mass-flow-rate. $\rho_{g}$ is defined as the density of air. $d_{c}$ is defined as the diameter of the cavitator. $U$ is defined as the water flow speed.

The reflux coefficient is gradually declined when approaching the nozzle outlet, which is similar to the nose ventilated supercavity. However, the reflux coefficient is much lower than the traditionally ventilated one, which shows that higher backflow velocity is generated by the reflux although the cavity shape looks similar. It leads to stronger 
impingement and higher turbulence on the head region. The reflux coefficient declines dramatically downstream of the nozzle outlet and achieves the minimum value near the vortex kernel location. It is indicated that most of the reflux gas recirculate in the vortex region of the nozzle outlet, and only a small portion is directed to the head region of the supercavity.

\subsubsection{The effect of jet flow rate on cavity scale and flow structure}

The flow patterns under different jet coefficients $\left(\bar{Q}_{j}\right)$ are analyzed in Fig. 15 . The length of the cavity gradually decreases as the jet coefficient continues to increase. The shedding mode at the closure would convert from the shedding of small bubbles to the oscillating deflation of large bubbles. Unexpectedly, the decrease of the supercavity number $\sigma_{c}$ is accompanied by the increase of the jet coefficient at some transient moments, which is in opposite trend with the ventilated supercavity. The supercavity number is $\sigma_{c}$ :

$\sigma_{c}=\frac{0.5\left(P_{\infty}-P_{c}\right)}{\left(\rho_{l} U_{\infty}^{2}\right)}$

Where $\rho_{l}$ is defined as the density of water. $P_{\infty}$ is define as the standard atmospheric pressure outside the water tunnel. $P_{c}$ is the transient pressure during the bubble shedding process from the main cavity. The transient pressure inside the cavity can be greater than the ambient pressure at shrinking moment, and the supercavity number may be negative. There is a positive correlation between the supercavity number $\sigma_{c}$ and the cavity length $\left(L_{c}\right)$.

When the reflux coefficient reaches a critical small value, the entire cavity will collapse. Comparing the reflux coefficient curve under different jet coefficients in Fig. 16, the maximum reflux coefficient increases with the raise of the jet coefficient. Only a small part of jet gas is re-directed into the head region to satisfy the cavity stability, while most of the gas still flows to downstream. The ventilated supercavity has a hysteresis effect in the process of cavity formation (Erfanian and Moghiman 2020), which dictates that the cavity could be sustained at much lower values of the ventilated coefficient than the amount required to form it.

Therefore, the reflux rate is equivalent to the required amount to sustain it after the initial supercavity is formed. In addition, it is also discovered that the reflux coefficient in the upstream of the nozzle outlet is roughly the same for the 2.73, 3.24 and 3.61 cases in Fig. 16. The location of the maximum backflow rate occurs almost coincides for the three cases, which implies that the same size of head cavity is maintained under the same reflux coefficient. When the closure position gets closer to the nozzle outlet, the maximum cavity scale is decreased and a weaker reflow is observed at nozzle

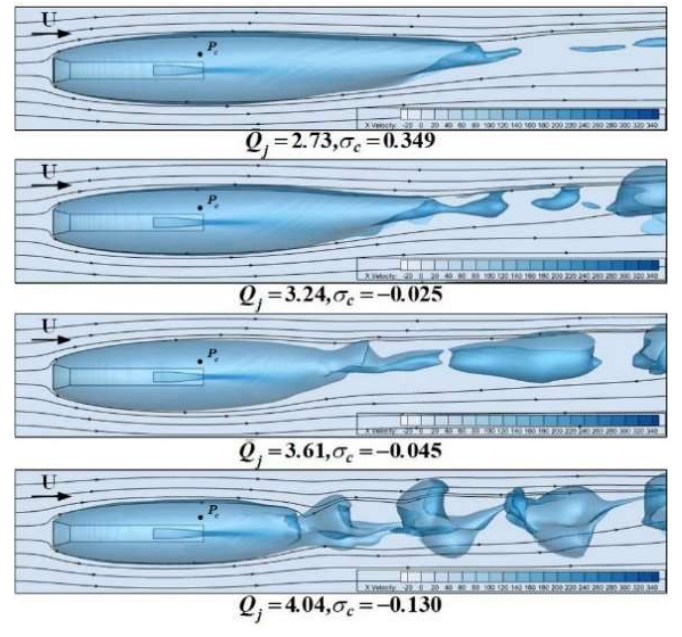

Fig. 15. Different cavity length $\left(L_{c}\right)$ corresponding pattern of jet coefficients $\left(\bar{Q}_{j}\right)$ and supercavity number $\left(\sigma_{\mathrm{c}}\right)$.

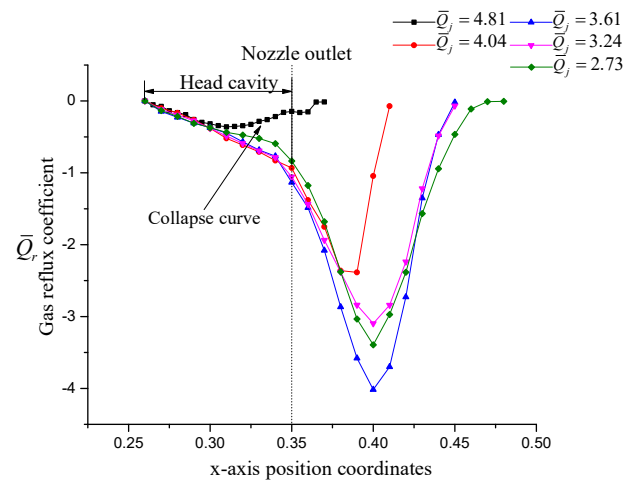

Fig. 16. Gas reflux coefficients of different reflux regions inside the supercavity.

tail. Obviously, due to the difference in the reflux coefficient of the nozzle outlet, the cavity scale is changed significantly. It is also found that the larger the jet coefficient $\left(\bar{Q}_{j}\right)$, the smaller the gas reflux coefficient $\left(\bar{Q}_{r}\right)$, and the smaller the cavity size $\left(\bar{L}_{c}\right)$. When the reflux coefficient reaches a critical small value, the entire cavity will collapse.

\subsubsection{Transient characteristics of jet-reflux supercavity}

As mentioned in Fig. 9, the jet-reflux supercavity shows different shedding scheme from nose ventilated supercavity. The cavity tail will take place series of "expansion, bulging, necking and broken up" processes. This will cause pressure fluctuation, cavity instability or even destruction. Therefore, the transient process of supercavity development is numerically simulated and analyzed with different jet intensity. Fig. 17 shows the change about pressure distribution during the bubble separation process from the main cavity, and the pressure 
pulsation is very obvious. From the first to the fourth picture in Fig. 17a, it can be seen that the pressure increases from 99452.6 to $102721 \mathrm{~Pa}$ near the outer boundary of the cavity. When the closure position of supercavity starts to shrink, the pressure inside the supercavity reaches the minimum. When the bubbles are about to fall off, the pressure will reach the maximum, which may lead to the tortuous cavity boundaries. Especially in the case of high jet coefficient, the tortuosity of the cavity is more obvious. As shown in Fig. 17b, the cavity boundary near the cavitator head is seriously deformed and is close to a collapsed state. Comparing the bubble shedding process under two different jet coefficients in Fig. 17, it is found that the larger jet velocity, the easier to form dispersed bubbles in the wake area, which is consistent with the observation in the experiment. In addition, under the condition of larger jet velocity, the closure location of the supercavity

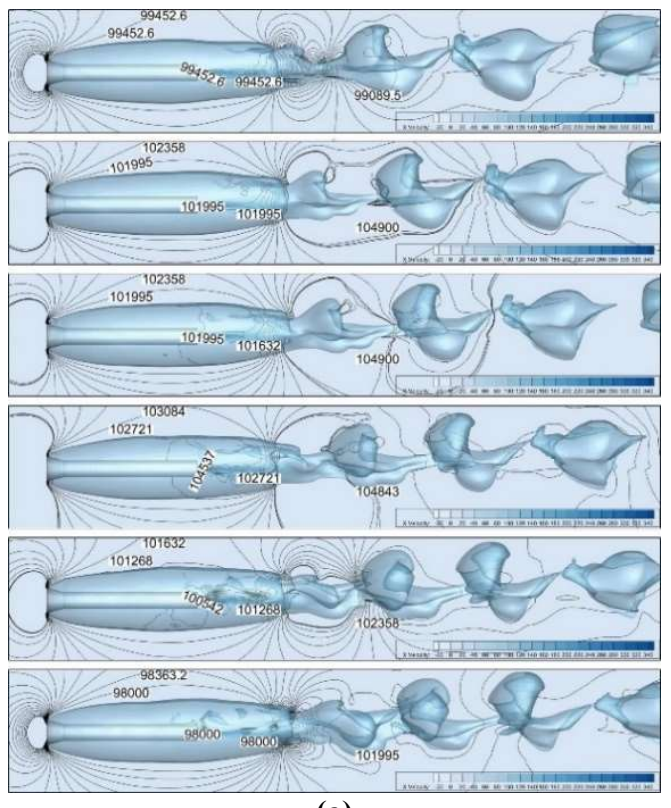

(a)

Fig. 17. Bubbles shedding morphology with an air iso-surface volume fraction of 0.5 and contour lines by static pressure in different jet coefficient (a) $\bar{Q}_{j}=4.04$ (b) $\bar{Q}_{j}=4.81$. shrinks to the nozzle outlet. The cavity interface is deformed obviously and the fluctuation is violent. It can also be seen that there are abundant of water droplets moving from the nozzle outlet towards the head of the cavity in Fig. 17b. Due to the higher reflux velocity (Fig. 16, $\bar{Q}_{j}=4.81$ ), the ambient liquid flow is entrained into the supercavity and then a large number of droplets are formed inside cavity. The cavity interface is impinged by the high-speed gas and liquid, which causes obvious deformation of the cavity interface. As a result, the tail jet flow could be used to generate supercavity to cover the whole vehicle. Yet, the stability of the supercavity would be reduced by the re-entrant jet liquid under the higher tail jet condition. In order to enhance the stability of the jet-reflux supercavity, it is advised to use some gas re-directed structure to reduce the reflux velocity.

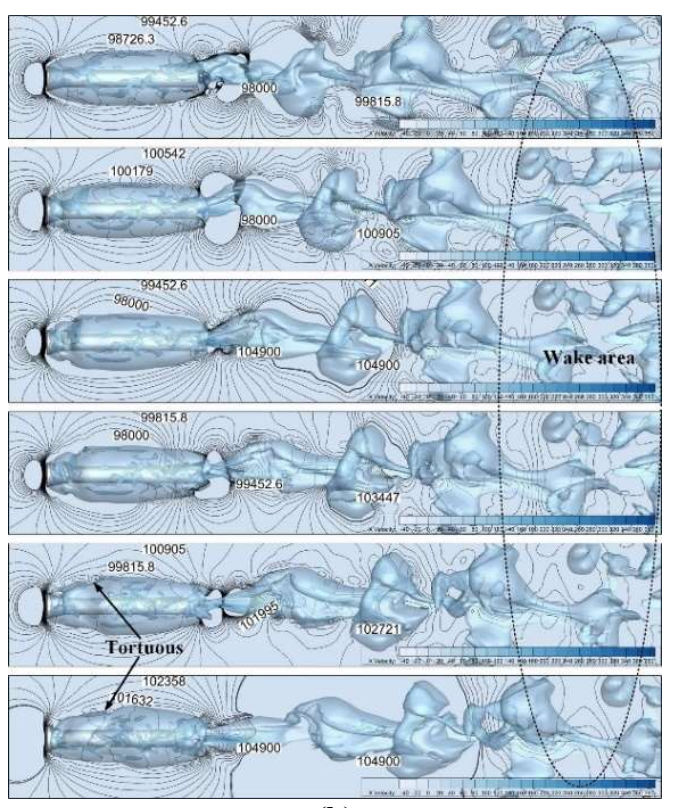

(b)

\section{CONCLUSION}

In this study, experiments and numerical simulations are combined to explore the formation process and entrainment characteristic of supercavity by the reflux of tail jet flow. Based on the open water tunnel, the experimental research can effectively reduce the air content in the water and improve the efficiency of the experiment. The high-resolution imaging technology is used to observe the jet-reflux supercavity. The formation and collapse conditions of the supercavity are explored by controlling the jet input and changing the scale of the revolution body. The main research contents and conclusions are as follows:

1) When an initial ventilated supercavity is formed to cover the jet outlet, the tail jet gas can quickly flow forward to maintain the integrity of the supercavity. Even when the nose ventilation is shut down, a stable cavity can still be formed. As the increase of the jet input, the supercavity transited from the twin-vortex coupling oscillation mode to purely bulging mode. Duo to the influence of buoyancy and internal pressure of cavity, the incline angle of two-vortex tube separation line $(\beta)$ increases as the closed position moving forward. The entire cavity presents axisymmetric flow state, while the ventilated supercavity appears to drift upward morphology. Moreover, the scale of the jet-reflux supercavity declines with the increase of jet coefficient. When the jet coefficient keeps increasing, the supercavity will collapse finally. Under the same jet coefficient, the scale of jet-reflux supercavity extends with the increase of the body length, while the collapse 
critical jet coefficient of the supercavity decreases with the increase of the body length.

2) Through numerical simulation analysis, the steep pressure gradient appeared near the nozzle outlet, and the streamline inside the jet-reflux supercavity presents considerable three-dimensional asymmetrical inflating flow characteristics in comparison with that of the ventilated supercavity. There is a huge recirculation zone between the nozzle outlet and the closure position of cavity. The variation of the gas reflux coefficient along the axial direction inside the supercavity is obtained. The maximum reflux coefficient occurs near the vortex kernel of the recirculation region. Only a small portion is directed to the head region of the supercavity, which indicated that the reflux rate is sufficient to satisfy the gas backflow inside the supercavity.

3) With different jet coefficient, similar scale of head cavity is sustained. When the supercavity closes near the nozzle outlet, a great number of droplets will be entrained into the cavity. The cavity interface will be impinged by the high-speed gas and mixture liquid, causing obvious deformation of the cavity interface. Finally, collapse will occur. In conclusion, the tail jet flow could be used to maintain supercavity and achieve drag reduction. However, the stability of the supercavity could be reduced by the re-entrant jet liquid under high jet input. In order to enhance the stability of the jet-reflux supercavity, it is advised to use some gas re-directed structure to control the reflux.

\section{ACKNOWLEDGMENTS}

The authors gratefully acknowledge support by the National Nature Science Foundation of China (NSFC, Grant NO: 52176164 and NO:51776221) and the Research Project Foundation of National University of Defense Technology (Grant NO:ZK18-02-07).

\section{REFERENCES}

Ahn, B. K., S. W. Jung, J. H. Kim, Y. R. Jung and S. B. Kim (2015). Experimental study on artificial supercavitation of the high speed torpedo. Journal of the Korea Institute of Military Science and Technology 18(3), 300308.

Balakrishna, A., S. Shao, Y. Liu and J. Hong (2019). Effect of Cavitator Geometry on the Ventilation Demand to Form and Sustain a Ventilated Supercavity. arXiv preprint arXiv:1903.10021.

Cameron, P. J., P. H. Rogers, J. W. Doane and D. H. Gifford (2011). An experiment for the study of free-flying supercavitating projectiles. Journal of Fluids Engineering 133(2), 021303.

Erfanian, M. R. and M. Moghiman (2020). Experimental investigation of critical air entrainment in ventilated cavitating flow for a forward facing model. Applied Ocean
Research 97, 102089. Retrieved from https://www.sciencedirect.com/science/article /pii/S0141118719310545.

Jiang, Y., T. Bai and Y. Gao (2017). Formation and steady flow characteristics of ventilated supercavity with gas jet cavitator. Ocean Engineering 142, 87-93.

Jihua, Z. B. Z. Y. Z. (2011). Experimental study on characteristics of ventilation supercavition generation and collapse. Chinese Journal of Applied Mechanics 28(1), 55-58.

Jin, D., C. Wang, Y. Wei, W. Cao, F. Yu and Z.-z. ZOU (2011). Experimental study of ventilated supercavity by underwater projectile. Engineering Mechanics 28(9), 214-217.

Karn, A. and S. Chawdhary (2018). On the synergistic interrelation between supercavity formation through vaporous and ventilated routes. International Journal of Multiphase Flow 104, 1-8.

Karn, A., R. E. Arndt and J. Hong (2016). An experimental investigation into supercavity closure mechanisms. Journal of Fluid Mechanics 789, 259.

Kawakami, E. and R. E. Arndt (2011). Investigation of the behavior of ventilated supercavities. Journal of Fluids Engineering 133(9), 57-64.

Kinzel, M. P., M. H. Krane, I. N. Kirschner and M. J. Moeny (2017). A numerical assessment of the interaction of a supercavitating flow with a gas jet. Ocean Engineering 136, 304-313.

Kinzel, M., M. Moeny, M. Krane and I. Kirschner (2015). Jet-Supercavity Interaction: Insights from CFD. Journal of Physics: Conference Series 656(1), 012133

Kirschner, I. N., N. E. Fine, J. S. Uhlman, D. C. Kring, B. J. Rosenthal, T. Gieseke, R. Kuklinski, A. Varghese, D. Stinebring, J. Dzielski (2001). Supercavitation research and development. Undersea Defense Technologies, Waikiki, HI, 1.

Lee, S. J., E. Kawakami and R. E. Arndt (2013). Investigation of the behavior of ventilated supercavities in a periodic gust flow. Journal of Fluids Engineering 135(8), 081301.

Menter, F. R. (1992). Performance of popular turbulence model for attached and separated adverse pressure gradient flows. AIAA Journal 30(8), 2066-2072.

Menter, F. R. (1994). Two-equation eddy-viscosity turbulence models for engineering applications. AIAA Journal 32(8), 1598-1605.

Menter, F. R. (2009). Review of the shear-stress transport turbulence model experience from an industrial perspective. International Journal Of Computational Fluid Dynamics 23(4), 305316. 
B. Liu et al. / JAFM, Vol. 15, No. 1, pp. 293-309, 2022.

Olsson, E., G. Kreiss and S. Zahedi (2007). A conservative level set method for two phase flow II. Journal of Computational Physics 225(1), 785-807.

Osher, S. and J. A. Sethian (1988). Fronts propagating with curvature-dependent speed: Algorithms based on Hamilton-Jacobi formulations. Journal of Computational Physics 79(1), 12-49.

Owis, F. and A. Nayfeh (2002). A compressible multi-phase flow solver for the computation of the supercavitation over high-speed torpedo. Paper presented at the 40th AIAA Aerospace Sciences Meeting and Exhibit.

Paryshev, E. V. (2006). Approximate mathematical models in high-speed hydrodynamics. Journal of Engineering Mathematics 55(1-4), 41-64.

Pearce, B. W. and P. A. Brandner (2012). Experimental investigation of a baseventilated supercavitating hydrofoil with interceptor. Paper presented at the Proceedings of the Eighth International Symposium on Cavitation-Cav2012.

Rashidi, I., Pasandideh-Fard, M., Passandideh-Fard, M. and Nouri, N. M. (2014). Numerical and Experimental Study of a Ventilated
Supercavitating Vehicle. Journal of Fluids Engineering 136(10), 101301.

Wang, W., Z. Zhang, G. He and W. Mo (2021). Numerical Analysis of the Effects of Periodic Gust Flow on the Wake Structure of Ventilated Supercavities. Journal of Marine Science and Application 20(1), 34-45.

Wang, Z., B. Huang, M. Zhang and G. Wang (2018). Experimental and numerical investigation of ventilated cavitating flow structures with special emphasis on vortex shedding dynamics. International Journal of Multiphase Flow 98, 79-95.

Xiang, M., X. Zhao and H. Zhou (2021). Transient dynamic analysis for the submerged gas jet in flowing water. European Journal of Mechanics-B/Fluids 85, 351-360.

Xu, C., J. Huang, Y. Wang, X. Wu, C. Huang and X. Wu (2018). Supercavitating flow around high-speed underwater projectile near free surface induced by air entrainment. AIP Advances 8(3), 035016.

Yi, W. j., T. h. Xiong and Y. X. Liu (2008). Numerical simulated research on characteristic of supercavity form around underwater high-speed projectile. Ship Science and Technology 4(1), 118-122. 